Research Article

\title{
Sports Information Acquisition and Functional Training System Based on Multisensor Information Fusion
}

\author{
Zhonglin Ma, ${ }^{1}$ Zhihao $\mathbf{Y u}^{1}$ and Jingshan Zhang $\mathbb{D}^{2}$ \\ ${ }^{1}$ College of Physical Education, Jilin Normal University, Siping, 136000 Jilin, China \\ ${ }^{2}$ Department of Sports Humanities, Sichuan Sports College, Chengdu, 610043 Sichuan, China \\ Correspondence should be addressed to Jingshan Zhang; zhangjingshan2007@126.com
}

Received 9 July 2021; Revised 5 August 2021; Accepted 16 August 2021; Published 2 September 2021

Academic Editor: Mu Zhou

Copyright (C) 2021 Zhonglin Ma et al. This is an open access article distributed under the Creative Commons Attribution License, which permits unrestricted use, distribution, and reproduction in any medium, provided the original work is properly cited.

\begin{abstract}
Since entering the 21st century, multisensor information fusion technology has developed rapidly, and multisensor information fusion is a trend in the coming years and even decades. This article is aimed at studying how the traditional sports information acquisition and functional training system can be implemented under the multisensor information fusion. This article proposes the use of multisensor information fusion technology to conduct an in-depth study on the traditional ways of acquiring sports information and the cognition of sports information by different groups of people, as well as the use of multisensor information fusion technology to update the functional training system and discuss whether this new functional training system is in line with the speed and rhythm of the current era compared with the traditional training system. The results of obtaining sports information from different groups of people show that more people know about sports events at home and abroad, reaching $22.5 \%$. Under the same circumstances, $60 \%$ of the elderly are willing to pay attention to sports information, while only $30 \%$ of the young people are willing to pay attention to sports information. And from 2016 to 2021 , the number of people in functional training nationwide has increased from 200,000 to 1.5 million.
\end{abstract}

\section{Introduction}

With the accelerated development of information and the Internet of Things era, information acquisition, comprehensive analysis, and processing of information and information fusion have become research hotspots in the field of information technology. Information system is our most important carrier for obtaining information resources, and it will inevitably become the information science main research object. The huge amount of information in the information system brings obvious uncertainty. There are many uncertainties in the source, true and false, and quality of the information. These uncertain information are also important topics in data mining and knowledge discovery. It can be seen everywhere in our daily life, which greatly affects all aspects of life, and is one of the very important social components. The Internet has become an important media tool for sports communication by virtue of its advantages such as rapid transmission, strong interactivity, fast release, and large amount of information. Especially since the inte- gration of the Internet, traditional media, and mobile phone new media, the network's advantages in sports communication have been exerted. Nowadays, people have attached great importance to daily sports. Online sports information is quietly affecting people's sports awareness, interest, attitude, and other sports awareness and changing people's sports behaviors such as participation in physical exercise, sports consumption, and acquisition of sports information. However, since the 2010s, various techniques have been combined with functional training, which has led to the prevalence of functional training in our country. Many scholars have proposed systematic research in theory; some have proposed functional training theories, and the proposed integration technology, coupled with a detailed combination method that fits our country's national conditions, has led to functional training in many places in our country.

The idea of multi-information fusion is like the human brain. It fully integrates the data information of multiple sensors. Through the reasonable analysis and use of the observed multidimensional information, the multidimensional sensors 
are spatially redundant or complementary. The information completes the fusion operation according to the established rules and finally obtains the desired combined result. The basic principles of multi-information fusion include the following: sensors in multiple dimensions receive the information of the target system, the information obtained by the sensor is processed with a certain algorithm, and finally, a data vector that can represent our needs is obtained; the output data of the sensor is used by the information fusion algorithm. According to specific rules, the mathematical description of the described system is obtained. And huge sports information resources. The Internet is constantly spreading vast and ever-changing sports information, and it provides uninterrupted reports on sports events and sports news. The speed of Internet communication is now developing rapidly. Compared with traditional media, Internet media has a big update in the amount of information and has strong interactivity. Now people's main source of information is from the Internet and various mobile terminal capabilities. It is more convenient and quicker than traditional methods to allow people to obtain information anytime and anywhere. Traditional media dissemination of sports information is a relatively single one-way transmission, while the network can present strong interactivity when disseminating sports information and it can spread in multiple directions. For example, in the live broadcast of a sports game, the audience can comment and exchange views in real time.

Multisensor information fusion (IF) has attracted the attention of many researchers in different fields because it can improve modeling accuracy by integrating information collected from multiple sensors. Traditionally, the input to multisensor IF problem is mostly quantitative data. However, in order to provide more comprehensive decision support, quantitative data and expert domain knowledge should be integrated in the IF process. In addition, there may be insufficient data in many practical situations, which makes many conventional methods unsuitable. Because the belief rule base (BRB) has advantages in nonlinear modeling with insufficient data and expert domain knowledge, the BRB-IF model is proposed for the multisensor intermediate frequency problem. Yu et al. proposed a specific optimization model for BRB-IF and its corresponding specific optimization algorithm, which can greatly improve efficiency. The model they proposed can test the optimization algorithm of particle swarms, so that particle swarms can be more efficient for activities, and differential evolution algorithm as an optimization engine, it can greatly improve the calculation speed of solving operator recommendation strategies. The effectiveness of the proposed BRB-IF is verified through actual case studies of threat level assessment, in which a comparison between BRB-IF and neural network is carried out. When training radial basis function neural network (RBFNN), the position of Gaussian neurons is usually determined by clustering. However, Yu et al. did not use a large amount of data information to conduct a comprehensive analysis and introduction of multisensor information fusion and finally got a convincing result [1]. The training input can be clustered in a completely unsupervised manner (input clustering), or some supervision can be introduced, for example, by concatenating the input vector with the weighted output vector (input-output clustering). Raitoharju et al. recommend applying clustering (class-specific clustering) to each class separately. This idea has been used in some previous work, but the benefits of this method have not been evaluated. When training RBFNN, we compared class-specific, input, and input-output clustering methods in terms of classification performance and computational efficiency. To achieve this goal, we applied three different clustering algorithms and conducted experiments on 25 benchmark data sets. The process of the experiment shows that the specific class-based method significantly reduces the overall complexity of clustering. The experimental results of Raitoharju et al. show that this method can significantly improve the classification ability and is widely used in functional training, fusion principles, etc., especially in terms of lack of Gaussian neuron network. Among other clustering algorithms, Raitoharju et al. combine dynamic evolutionary optimization, multidimensional particle swarm optimization, and specific clustering to optimize the number and location of cluster centroids for the first time. However, Raitoharju et al. did not make a summary explanation with their experimental results in the functional training systems, leading to their theory may still be insufficient [2]. Raquel et al. used distributed and centralized fusion methods to study the problem of information fusion estimation for a class of multisensor linear systems affected by different types of random uncertainties. It is assumed that the measurement output is disturbed by one-step autocorrelation and crosscorrelation additive noise, and Raquel et al. also consider the random uncertainty caused by multiplicative noise and random loss of measured values in the sensor output. At each sampling time, each sensor output is sent to the local processor, and due to some kind of transmission failure, a step-related random delay may occur. Only the covariance information is used, and the evolution model of the signal process is not required. The local minimum squares (LS) filter based on the measured value received from each sensor is designed through an innovative method. Then, use the LS optimality criterion to fuse all these local filters through matrix-weighted linear combination to generate the best distributed fusion filter. In addition, a recursive algorithm of centralized fusion filter is proposed, and the accuracy of the estimator measured by the estimation error covariance is analyzed through simulation examples. However, Raquel et al. did not conduct an analysis of the uncertain multisensor information fusion technology to support their experimental $\operatorname{logic}[3]$.

The research on functional training in this article is carried out on the basis of multi-information fusion and uses the latest network, communication, 5G, and other technologies. Network technology can control the transmission of information in the local area network, and communication technology can enable information to be transmitted wirelessly and can be very good for the acquisition of sports information and the realization of related functional training. It can also be said to be multisensor information convergence. It is the correlation, fusion, and update of specific 


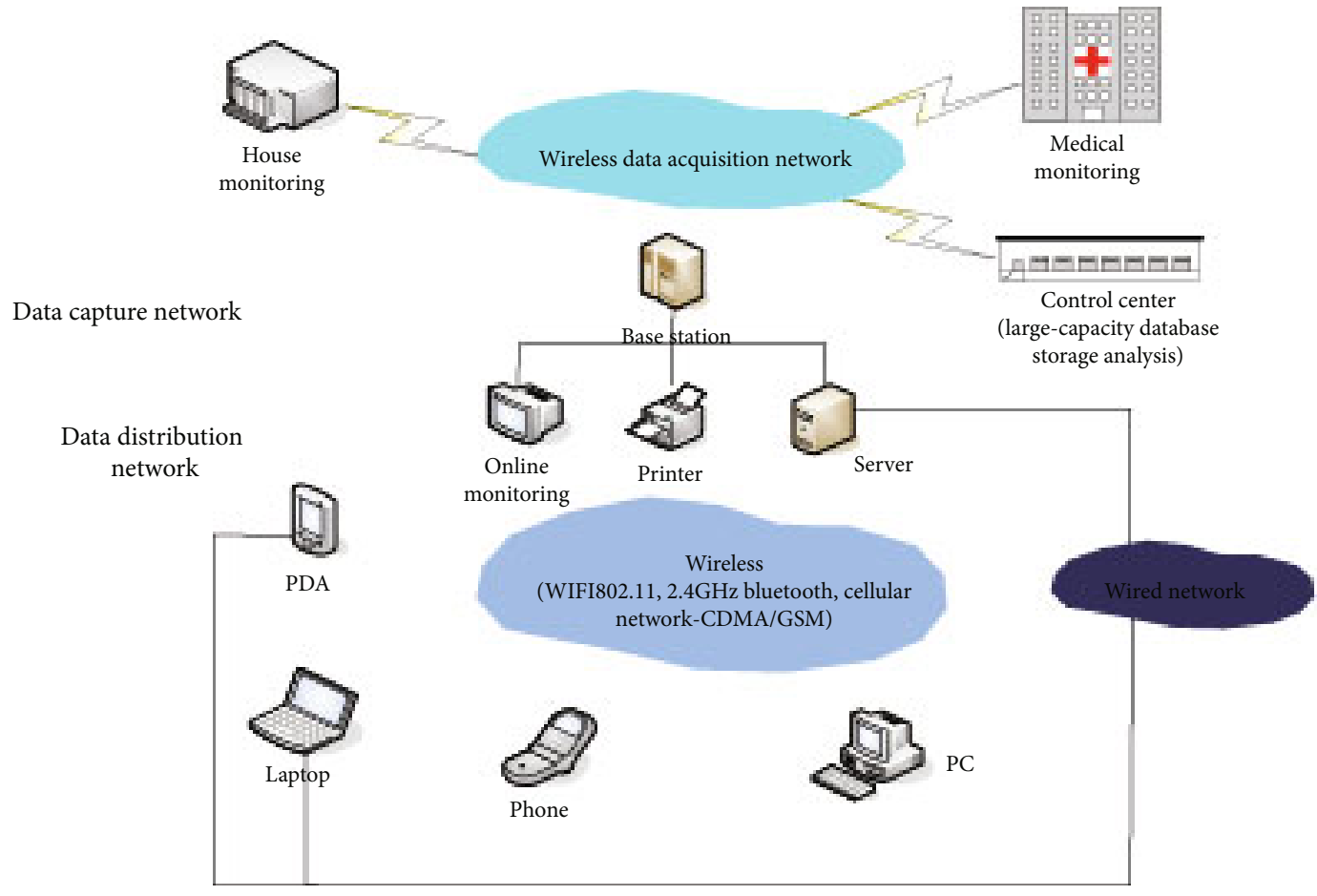

FIGURE 1: Comprehensive map of multisensor information fusion.

experimental data such as data core information that people obtain from multiple different information sources to achieve acquisition, very accurate location, and corresponding identity estimation, including the processing of information obtained from a very comprehensive assessment of the situation and threats and their importance. This process is a continuous process of estimating and evaluating the needs of other information sources. At the same time, it is also a process of continuous self-correction of information in the process of processing and finally gets the results you want. At present, people's acquisition of sports information may still remain in the traditional and single method, including traditional media and the current network. Traditional techniques are also used when performing functional training, which has not kept up with the changes in this era. On the basis of traditional theory, this article uses new multiinformation fusion technologies, as well as the Internet, 5G, and communication technologies to have a new way to obtain sports information, while the traditional functional training is updated. The previous methods used more scientific and practical methods and methods for training.

\section{Multisensor Information Fusion Method and Information Acquisition}

2.1. Multisensor Information Fusion Technology. The socalled multisensor information fusion (MSIF) is the use of computer technology to automatically analyze and synthesize information and data from multiple sensors or sources under certain criteria to complete the required decisions and estimates [4]. Multisensor information fusion is an information processing technology used to include multiple sensors in different locations, as shown in Figure 1. In the mature development of modern sensor technology, data processing technology, computer software and hardware technology, and industrialized control technology, data processing technology can process the information collected by sensors correspondingly, and computer software and hardware can play a basic role in the transmission process. Industrialization of the control can carry out the control operation of the sensor, and now the multi-information sensor information fusion technology has become a very popular technology. Nowadays, my country's related research on sensor information fusion technology has been used in ordinary engineering to locate and identify information [5]. The positioning of information can accurately locate the products produced, and subsequent maintenance updates and identification technology have been carried out and can effectively identify relevant information. And it is believed that with the advancement of science, multisensor information fusion technology will become a specialized technology for comprehensive processing and research of intelligent and refined data, information, and images [6].

It can be obtained from the statistical method of probability theory. Information entropy is the average uncertainty of the information source. It is defined in Shannon information theory as the elimination or reduction of people's understanding of uncertain things. This method can be achieved through sensor technology. The purpose of collecting and fusing information can provide good statistics on the information transmitted [7]. According to the principle of information theory, the information content of multidimensional information fused by single-dimensional information is greater than that of any single-dimensional information. 
This is the purpose of multisensor information fusion [8]. The proof is as follows:

Because the entropy $H(X)$ of the random variable $X$ can only be a function of its probability distribution $p_{1}$, $p_{2}, \cdots, p_{n}$, it is also recorded as follows:

$$
H(X)=H\left(p_{1}, p_{2}, \cdots, p_{n}\right) \cong-\sum_{i=1}^{n} p_{i} \log p_{i} .
$$

In Equation (1), $0 \leq p_{i} \leq 1$, so

$$
H\left(p_{1}, p_{2}, \cdots, p_{n}\right) \geq 0
$$

If and only if each item is $-P_{I} \sum \log p_{i}=0(i=1,2, \cdots, n)$, the equal sign is true, namely, $p_{i}=0$ or $p_{i}=1$. And because of $\sum_{i=1}^{n} p_{i}=1$, if and only if $p_{i}=1, p_{k}=0(k \neq i, k=1,2, \cdots, n)$, the equal sign of Equation (2) holds [9].

Supposing the two sets of random variables are $X$ and $Y$ and the information entropy is $H(X)$ and $H(Y)$, respectively, then there is an additivity of information entropy to obtain the information entropy of the joint set $X Y$ :

$$
H(X Y)=H(X)+H(Y \mid X)
$$

Supposing the probability distribution of events in the $X$ set is $p_{1}, p_{2}, \cdots, p_{n}$, the conditional transition probability between the $X$ set and the $Y$ set is $p_{i j}$, and Equation (3) is expressed in the form of Equation (1), the information entropy of the two-dimensional random variable can be obtained as follows:

$$
\begin{aligned}
& H\left(P_{1} P_{11}, P_{1} P_{12}, \cdots, P_{1} P_{1 n}, P_{2} P_{21}, P_{2} P_{22}, \cdots, P_{2} P_{2 n}, \cdots, P_{m} P_{m 1}, P_{m} P_{m n}\right) \\
& \quad=H\left(p_{1}, p_{2}, \cdots, p_{n}\right)+\sum_{I=1}^{m} P_{I} H\left(p_{i 1}, \cdots, p_{i m}\right) .
\end{aligned}
$$

Among them,

$$
\sum_{j=1}^{m} p_{i j}=\sum_{j=1}^{m} p_{i 1}+p_{i 2}+\cdots+p_{i m}, \quad i=1,2, \cdots, n .
$$

From Equation (2) and Equation (4), we can get the following:

$$
\begin{aligned}
& H\left(P_{1} P_{11}, P_{1} P_{12}, \cdots, P_{1} P_{1 n}, P_{2} P_{21}, P_{2} P_{22}, \cdots, P_{2} P_{2 n}, \cdots, P_{m} P_{m 1}, P_{m} P_{m n}\right) \\
& \quad \geq H\left(p_{1}, p_{2}, \cdots, p_{n}\right) .
\end{aligned}
$$

When $N$ is a random variable $X_{1}, X_{2}, \cdots, X_{n}$, according to the additivity of information entropy, we can get the following:

$H\left(X_{1}, X_{2}, \cdots, X_{n}\right)=H(X)+H\left(X_{1} \mid X_{2}\right)+\cdots+H\left(X_{n} \mid X_{1} X_{2}, \cdots, X_{n-1}\right)$.

From Equations (6) and (7), it can be obtained that the information content of multidimensional information is greater than that of any single-dimensional information [10].

2.2. Sports Information Acquisition. At present, most people generally obtain sports information by some traditional methods, for example, through traditional media methods such as the Internet, TV, and newspapers. Such methods are very simple and require a large amount of data and support and require a lot of media to follow closely to obtain information [11]. The sports information acquisition based on the multisensor information fusion method we studied is completely different from the traditional method, and it is also very suitable for this fast-paced era [12]. The flow chart of obtaining sports information is shown in Figure 2:

CoreConnect is an integration technology developed and designed by IBM. It can connect many different chips to form a whole, and use this technology to reuse integration and core applications. In this experiment, we use this technology and the system can not only save costs but also improve system performance to a certain extent [13]. The CoreConnect bus contains its own bus (PLB), on-chip peripheral bus (OPB), 1 bus bridge, 2 arbiters, and 1 device control register (DCR) bus. The kernel needs to go through the OPB to access other system resources. Although the OPB bus is a synchronous bus, it is actually in an independent bus layer, providing unrelated 32-bit address bus and 32-bit data bus $[14,15]$. Moreover, it is not directly connected to the core to connect to the processor core; the core can access peripherals through the "PLB to OPB" bridge, and the peripherals can access the memory through the "OPB to PLB" bridge. The logical structure of the self-designed IP core is shown in Figure 3, which includes the NAND FLASH control part, DMA high-speed data storage part, sensor data transmission part, and USB control part [16].

The three-axis accelerometer and the three-axis gyroscope in the MIMU sensor are composed of three orthogonal coordinate axes. The three measurement axes have different measurement properties, and the three measurement axes cannot guarantee two-by-two orthogonally [17]. The zero error can be expressed as follows:

$$
g_{0}=\left[g_{x 0} g_{y 0} g_{z 0}\right]^{\mathrm{T}}
$$

Among them, $g_{x 0} \quad g_{y 0} \quad g_{z 0}$ is the zero deviation of the $x$-axis, $y$-axis, and $z$-axis, respectively [18].

The sensitivity error is mainly caused by the different characteristics of the signal amplifier circuit on the three axes of the sensor.

$$
\begin{array}{rrrrrrrrr}
1 & 0 & 0 & \partial S_{x} & 0 & 0 & S_{x} & 0 & 0 \\
S=S_{n} 0 & 1 & 0+ & 0 & \partial S_{y} & 0 & = & S_{y} & 0 \\
0 & 0 & 1 & 0 & 0 & \partial S_{z} & 0 & 0 & S_{z}
\end{array}
$$

Among them is the rated sensitivity of the MIMU sensor, and $S_{x}, S_{y}, S_{z}$ is the sensitivity of the three axes [19].

The three coordinate axes of the MIMU sensor are completely orthogonal, but the three axes cannot be 


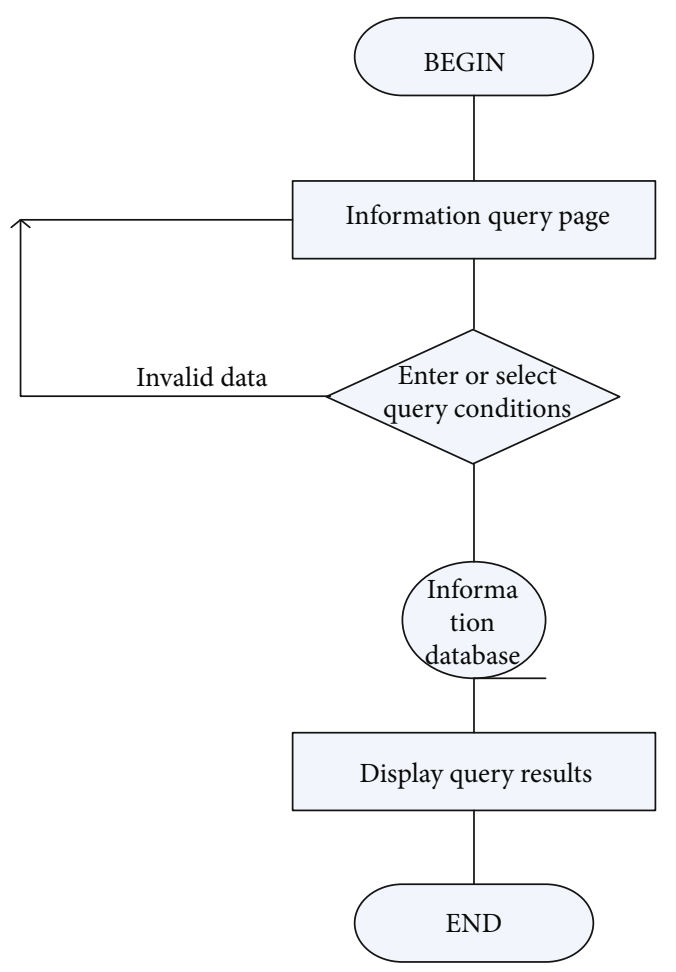

FIGURE 2: Flow chart of exercise information acquisition function.

guaranteed to be orthogonal in the sensor production process, resulting in nonorthogonal errors [20]. The nonorthogonal error can be expressed as follows:

$$
\begin{array}{cccccc}
\cos \alpha & 0 & \sin \alpha & 1 & 0 & \alpha \\
C=\sin \beta \cos \gamma & \cos \beta \cos \gamma & \sin \gamma \approx \beta & 1 & \gamma . \\
0 & 0 & 1 & 0 & 0 & 1
\end{array}
$$

Generally, the nonorthogonal error angle of the MIMU sensor provided by the manufacturer is less than 0.5 , so Equation (10) can be simplified [21]. Considering the above errors of the acceleration sensor, the true value $g_{e}$ and the measured value $g_{m}$ are as follows:

$$
g_{m}=S \cdot C \cdot g_{e}+g_{0}=D \bullet g_{e}+g_{0}
$$

Among them,

$$
\begin{aligned}
& \begin{array}{lll}
S_{x} & 0 & \alpha S_{x}
\end{array} \\
& D=\beta S_{y} \quad S_{y} \quad \gamma S_{y} . \\
& \begin{array}{lll}
0 & 0 & S_{z}
\end{array}
\end{aligned}
$$

Then,

$$
g_{e}=E\left(g_{m}-g_{0}\right)
$$

Among them,

$$
\begin{array}{rcc}
\frac{1}{S_{x}} & 0 & -\frac{\delta}{S_{z}} \\
E=A^{-1}=-\frac{\gamma}{S_{x}} & \frac{1}{S_{y}} & \frac{\beta \gamma-\vartheta}{S} . \\
0 & 0 & \frac{1}{S_{z}}
\end{array}
$$

Therefore, by calculating the zero error $g_{x 0}, g_{y 0}, g_{z 0}$, the sensitivity error $S$, and the nonorthogonal error angles $\alpha, \beta$, and $\gamma$, the measured value can be accurately calibrated to obtain the true value [22].

Ideally, the measured value $A$ satisfies the following equation:

$$
A^{T} \cdot A=(1 G)^{2} .
$$

That is, the acceleration modulus $\sqrt{\left(g_{x}\right)^{2}+\left(g_{y}\right)^{2}+\left(g_{z}\right)^{2}}$ of an ideal three-axis acceleration sensor in any posture is a fixed value $g$. The trajectory of the measured value $A$ is a gravitational spherical surface with a radius of $1 \mathrm{~g}$. Actually, due to various errors in the three axes of the acceleration sensor, the output trajectory will be an ellipsoid whose center deviates from the origin of the coordinates [23].

2.3. Functional Training System. After entering the 2010s, functional training entered our country and began to spread widely. Until now, although everyone has known that this is very beneficial for human training and recovery, the parties have not yet reached a consensus on the definition of a functional training system [24]. Gary Cook (1997) first put forward the concept of functional training. Functional training is an increase process that gradually improves athletic performance. At the very beginning, a model with high standard and high quality should be established. Firstly, it should correct poor posture and accelerate recovery from injuries. On this basis, the basic abilities of the human body, such as strength, speed, and balance, are further developed and repaired, and then, targeted repairs are carried out according to the different injury parts of different people. In the development of different movements according to specific abilities, the whole is similar to a pyramid [25]. The starting point of active-duty training is the mode of action. It can combine various functions by practicing human gestures and motion patterns in order to optimize the most basic exercise ability of the human body. Through functional spine strength energy chain recovery and regeneration, speed, explosive power, energy system, etc., it is used for systematic training and continuously strengthening the method system of cultivating sports talents. Execution system is the basic content of on-duty training. Studies have shown that healthy people have some limitations in completing their course of action. The main goal of functional training is to improve its implementation [26]. In daily life, it is necessary 


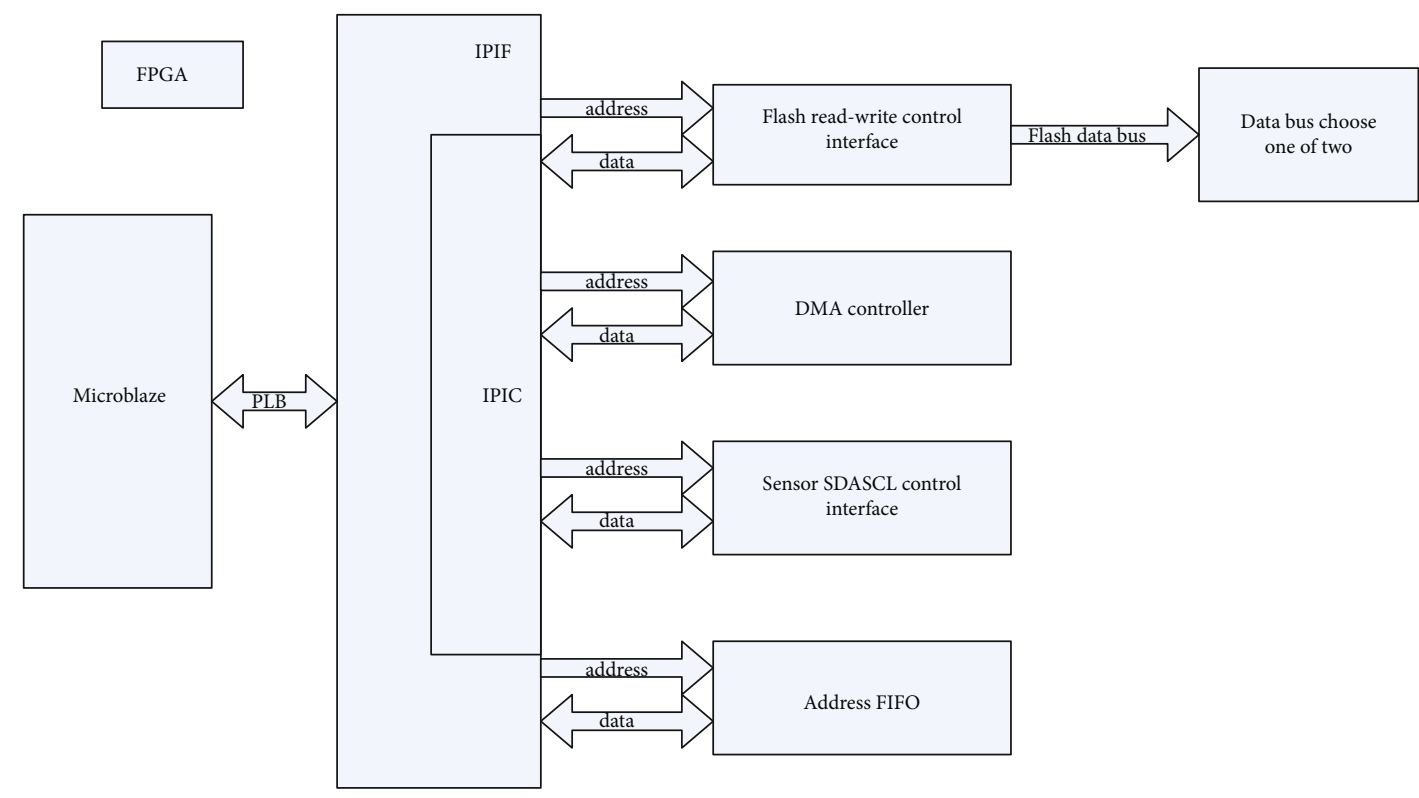

Figure 3: Internal logic diagram of self-designed IP core.

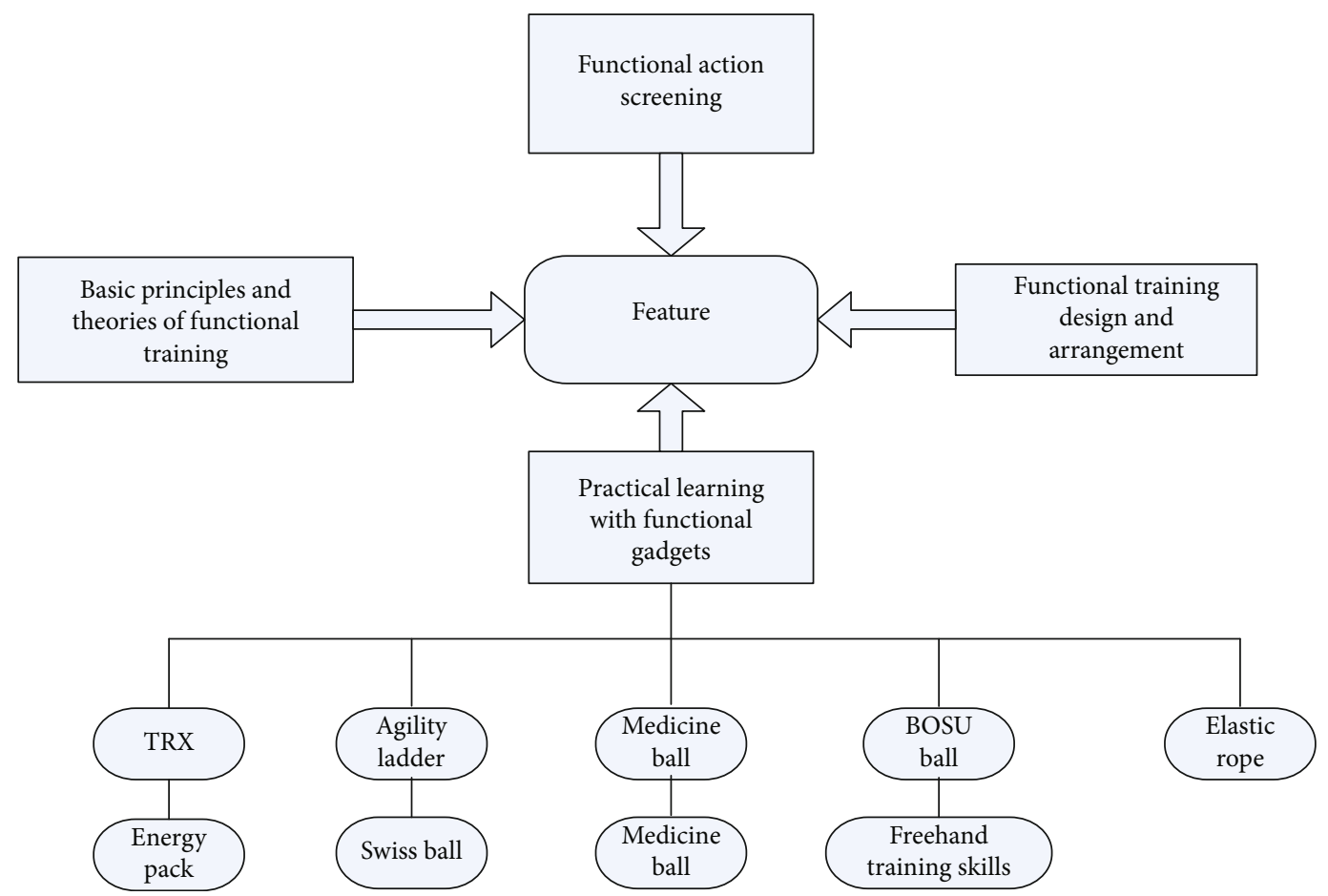

FIgURE 4: Detailed schematic diagram of the logical relationship of functional training.

to warm up and perform detailed system training and to ensure that you are not injured; you must train before you can enhance your physical fitness. Functional training is based on the principle of first evaluation and practice before you can carry out good training to improve our physical capabilities. In competitive sports, it seems to improve the ability of competitive athletes [27], as shown in Figure 4.

\section{Multisensor Information Fusion Platform}

3.1. Hardware Design. STMicroelectronics has developed the STM32 series of microarithmetic controllers. Its embedded application software system design has the advantages of high performance, low cost, and low power consumption. It has 32 bits developed based on the ARM Cortex-M3 core. Microcontroller uses the software in the experiment to 


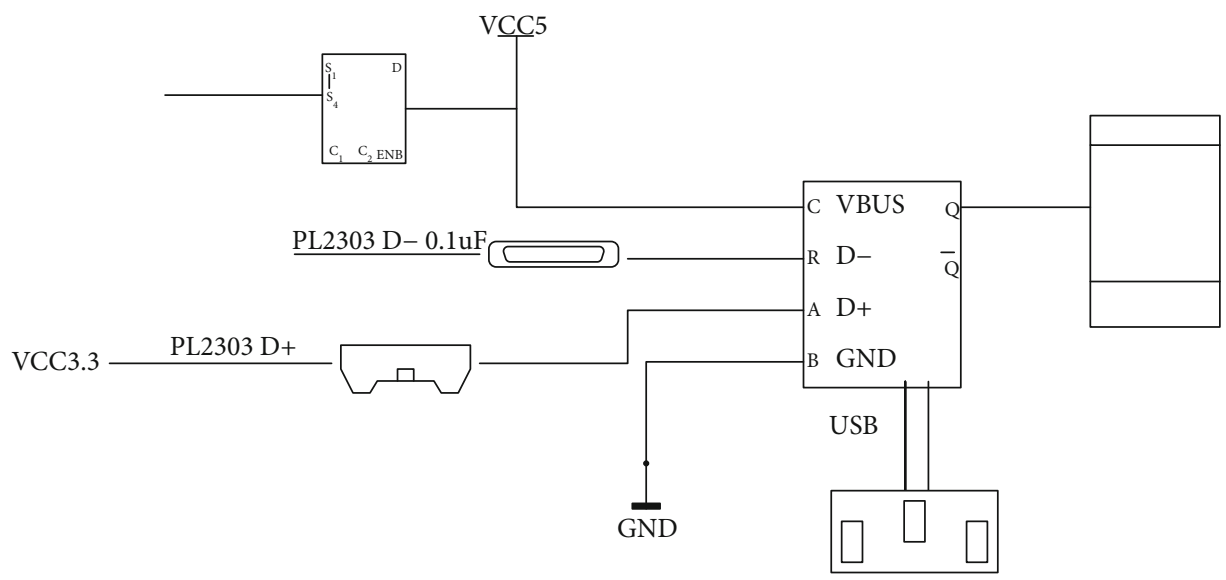

FIgURE 5: Detailed design drawing of the power circuit.

perform calculation and storage functions of related information. The power consumption of the code read and executed from flash is only $36 \mathrm{~mA}$, which is equivalent to $0.5 \mathrm{~mA} / \mathrm{MHz}$. Compared with all 32-bit microprocessors on the market, the power consumption of STM32F103 is the lowest. At the beginning of the design, the serial port was designed to communicate with the PC. Since the notebook is more convenient to carry and easy to debug, and the PC does not have a serial port, it is designed to use USB power supply. This power supply mode is convenient, simple, and fast, compared to the conventional power supply mode which has great flexibility. Considering that the power cord needs to be tightly connected to the development board when power is supplied, and cannot be loosened, based on this principle, the USB interface type at the PCB board adopts the way of USB to square port. The USB power supply circuit is shown in Figure 5. This method is relatively simple and fast and can obtain a stable $5 \mathrm{~V}$ voltage directly from the USB. If the $5 \mathrm{~V}$ voltage is self-made, the power supply voltage may not be stable enough due to the operation of the manufacturing process or the device. In addition, at the beginning of the design, it was considered that the power consumption of all devices when working at the same time was less than $2 \mathrm{~W}$, and the maximum power of USB power supply reached $2.5 \mathrm{~W}$, which can meet the power consumption requirements of the system of $3.3 \mathrm{~V}$ power supply requirements of some devices.

3.2. Software Design. This article uses the USB interface protocol as the communication interface between the system and the PC and sends command codes to the system through the PC to control system formatting, acquisition, and configuration parameters. The most important thing is to save the data collected by the system to the PC for analysis. This system uses FIDI's high-speed FT256 chip as the control chip for USB communication. The USB read and write control signals DR and WR and RXF and TXE status signals are directly connected to the FPGA chip. Directly address the registers through the MicroBlaze soft core, control the read and write modules, and read the status of RXF and TXE. The MicroBlaze soft core in the FPGA performs data reading and writing by controlling the coordination of various modules. There is a FIFO buffer area inside the USB chip. When RXF is low, it means that the FIFOreceiving buffer has received a byte of data from the host computer. When the $\mathrm{RD}$ read signal arrives at a low level, The FIFO controller in the chip will release the data bus. The FIFO data is released to the 8-bit data bus. At this time, the data on the bus is transferred to the soft core through the PLB bus, and the data at the 28PC end is transmitted to the system. After that, the RD signal is pulled high, and the RXF signal is also pulled high, indicating that the FIFO-receiving buffer is already empty and waiting for new data to be added.

3.3. Multisensor Information Fusion Platform. This article is based on the fusion of multisensor information to obtain traditional sports information and conduct an in-depth study on the way of functional training. Multisensor information fusion (MSIF) technology uses the latest computer science and technology to obtain data and information from a large number of ordinary sensors under certain rules or guidelines. The rules and guidelines include sensor technology, fusion principles, and information transmission. The rules are established by the parties. Information or data is researched or comprehensively analyzed to finally achieve the real-time information processing process for the desired results and decision-making. With the gradual development and maturity of various sensor application technologies, computer software and hardware technologies, and data information processing technologies since the 21st century, multi-information sensing technology has become a very popular technology in recent years. It is also very popular. Place multiple ordinary sensors in different locations to perform operations such as data perception, collection, processing, and the ability to give feedback to the processed information to the background for subsequent operations. This technology is called multi-information sensing technology. The technology can collect information operation because there are many different sensors placed in different locations. With the gradual deepening of science and technology, multi-information sensor fusion technology will be more and more in-depth research, and in the future, it will surely become a professional technology that integrates refined data, intelligent information, and image processing. 
However, the multisensor information fusion platform is also an immature platform technology. The multi-information fusion system is also a complex system with very strong uncertainty. The processing method of the platform has also received existing technology, equipment, theoretical knowledge, and limits. Although this is a newly developing subject, there are still many theoretical knowledge shortages. However, with the continuous development of science and technology and related subjects in the future, I believe that we will gradually come into contact with it and experience it in our future study and life to the convenience it brings us.

\section{Analysis and Discussion}

4.1. Data Analysis Based on Multisensor Information Fusion. Relying on the multisensor information fusion platform, this paper uses multisensor information fusion technology to study the acquisition of traditional sports information and functional training. Among them, we have done some questionnaires for the acquisition of sports information. Information is a survey and research conducted on different people of different ages and classes within the whole school. People's motivation for contact is shown in Table 1. People's motivations for acquiring sports information include increasing sports knowledge, understanding domestic and foreign sports competitions, studying, enjoying sports, wanting to improve conversation skills, leisure and entertainment, coping with exams, passing time, and others.

There are commonalities among different individuals, but there are also personalities between them. Differences in gender, age, and occupation affect the ways and motivations of different people to contact sports information. Table 2 shows that gender differences make different groups of people have great differences in their exposure to sports information. In terms of increasing sports knowledge, understanding domestic and foreign sports competitions, and enjoying sports, men far surpass women. However, in terms of learning and leisure and entertainment, there are far more women than men in dealing with exams and so on. People of different ages also have different purposes for acquiring sports information. The younger ones are mostly motivated to improve communication skills, leisure and entertainment, and pass time, while the older ones are to increase their sports knowledge and understand domestic and foreign sports competitions. Wait. The level of income also affects the motivation of obtaining sports information to a certain extent. Those with high income have slightly lower motives than those with lower incomes except for understanding domestic and foreign sports events, learning, and other motives, and other motives are higher than those with lower incomes. People with low levels rely more on sports information to meet their own needs.

In daily life, people have different ways to obtain sports information, including newspapers, television, Internet, and radio, and the types of sports-related information obtained based on different ways are also diverse. According to the different functions and attributes of the media, the sports information obtained is roughly divided into news, entertainment, and knowledge. There are six types of people
TABLE 1: People's motivation for obtaining sports information.

\begin{tabular}{lcc}
\hline & Number of people & $\%$ \\
\hline Increase sports knowledge & 1085 & 21.3 \\
Understand domestic and foreign & 1145 & 22.5 \\
sports competitions & 821 & 16.0 \\
Learn & 395 & 7.7 \\
Love sport & 263 & 5.1 \\
Want to improve conversation skills & 956 & 18.7 \\
Leisure and entertainment & 204 & 4.0 \\
Overcome exam & 196 & 3.8 \\
Kill time & 35 & 0.6 \\
Others & & \\
\hline
\end{tabular}

getting more sports information: news, entertainment, knowledge, comprehension, education, and comment. Our research is based on specific data obtained on this basis. The details of the data are shown in Table 3.

Sports information cognition means that sports participants have a systematic understanding and understanding of some basic sports and common knowledge. With more and more knowledge and understanding, they have a higher level of awareness of sports. The promotion function makes it easier to obtain detailed sports information, which is also of great benefit to the formation of sports behaviors. As shown in Figure 6, when conducting surveys and researches on people at various stages, it was found that different groups of people have different degrees of agreement on the cognitive impact of sports information, but in general, sports information has a significant impact on the sports of most people. The increase of sports knowledge and the understanding of sports have a very obvious effect; that is, obtaining sports information helps different people's cognition of sports.

The gradual improvement process of actual sports performance is fast and standardized. A cumulative process that gradually increases with the performance in the experiment is called functional training. At the very beginning, a standard and high-quality model is established instead of starting from the basics. Establishing a high standard for experimentation can achieve an expected experiment purpose. First, correct poor posture and accelerate recovery from injuries. On this basis, it is necessary to improve the basic abilities of the human body, such as strength, speed, balance, which are further developed and repaired, and then, targeted repairs are carried out according to different injured parts of different people. Different actions are developed according to specific abilities. The whole is similar to a pyramid. The basic action patterns of the human body are shown in Table 4 . When receiving different information, the feedback of the human body's perception in the brain is reflected in different areas.

In recent years, as the number of exercisers has increased year by year, the number of injured people has also increased year by year. Excluding professional athletes, in daily learning exercises, failure to master the correct exercise methods can easily cause injuries, o professional functions are important and the most basic. Sex training becomes very 
TABLE 2: Motivation of different groups of people to contact sports information.

\begin{tabular}{|c|c|c|c|c|c|c|}
\hline Motivation & Male & Female & Old & Young & Low & High \\
\hline Increase sports knowledge & 54.8 & 39.4 & 52.0 & 42.9 & 47.2 & 46.7 \\
\hline Understand domestic and foreign sports competitions & 63.4 & 36.3 & 54.9 & 45.5 & 47.8 & 52.1 \\
\hline Learn & 33.2 & 38.5 & 38.9 & 30.0 & 40.0 & 34.4 \\
\hline Love sport & 26.8 & 8.3 & 19.9 & 15.7 & 17.1 & 17.8 \\
\hline Want to improve conversation skills & 11.6 & 10.7 & 9.0 & 12.1 & 12.3 & 8.5 \\
\hline Leisure and entertainment & 56.9 & 60.2 & 56.0 & 60.1 & 62.6 & 53.5 \\
\hline Overcome exam & 5.5 & 11.4 & 8.1 & 8.7 & 10.1 & 6.3 \\
\hline Kill time & 19.2 & 27.0 & 19.0 & 26.0 & 23.7 & 22.5 \\
\hline Others & 0.7 & 0.7 & 0.41 & 0.9 & 0.5 & 1.0 \\
\hline
\end{tabular}

TABLE 3: Types of people getting more sports information.

\begin{tabular}{lcccccc}
\hline & News & Entertainment & Knowledge & Comprehension & Education & Comment \\
\hline Number & 1366 & 1427 & 890 & 708 & 478 & 514 \\
$\%$ & 59.4 & 62.1 & 38.7 & 30.8 & 20.8 & 22.3 \\
Sorting & 2 & 1 & 3 & 4 & 6 & 5 \\
\hline
\end{tabular}

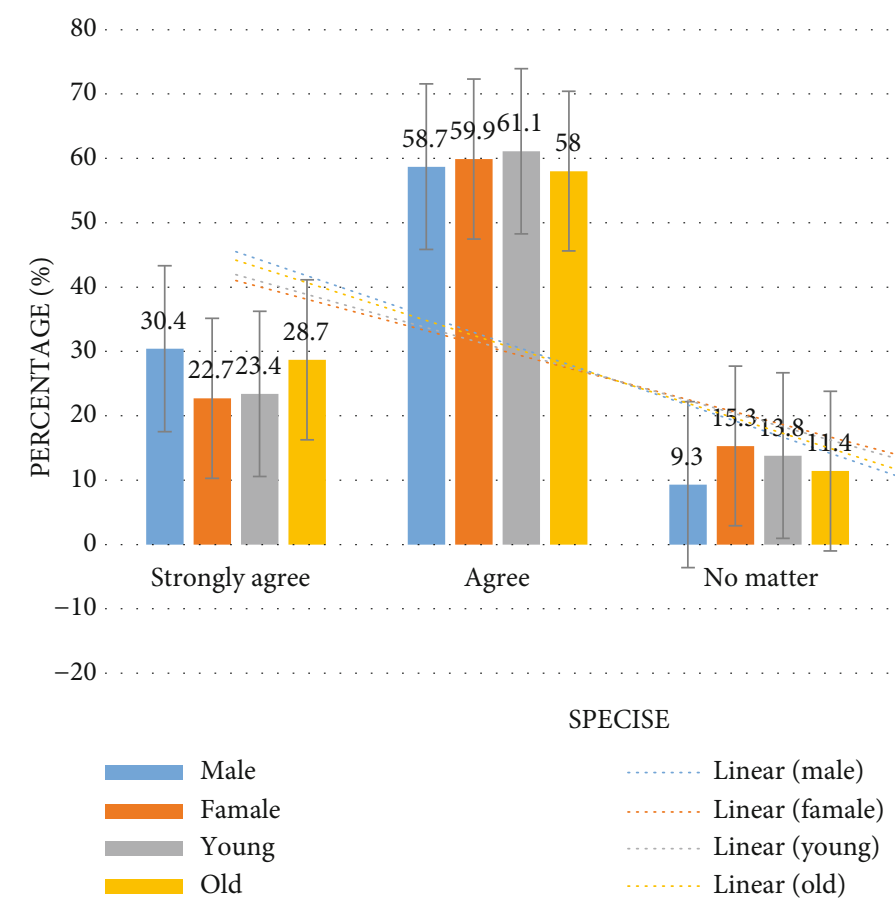

FIgURE 6: The influence of obtaining sports information on sports cognition of different groups of people.

TABle 4: Patterns of basic human movements.

\begin{tabular}{lcc}
\hline Basic action mode & Daily life example & Examples of fitness life \\
\hline Squat & Sit and stand & Weight-bearing squat exercise \\
Lunge & Go up the stairs & Lunge walking practice \\
Gait & Walking or running & Rhythm running \\
Body flexion & Bent over to pick up things & Back lifting exercises \\
Body rotation & Turn around to take something & Upper body diagonal pull exercise \\
Push support & Push the door or prop up the body & Push-up exercises \\
\hline
\end{tabular}




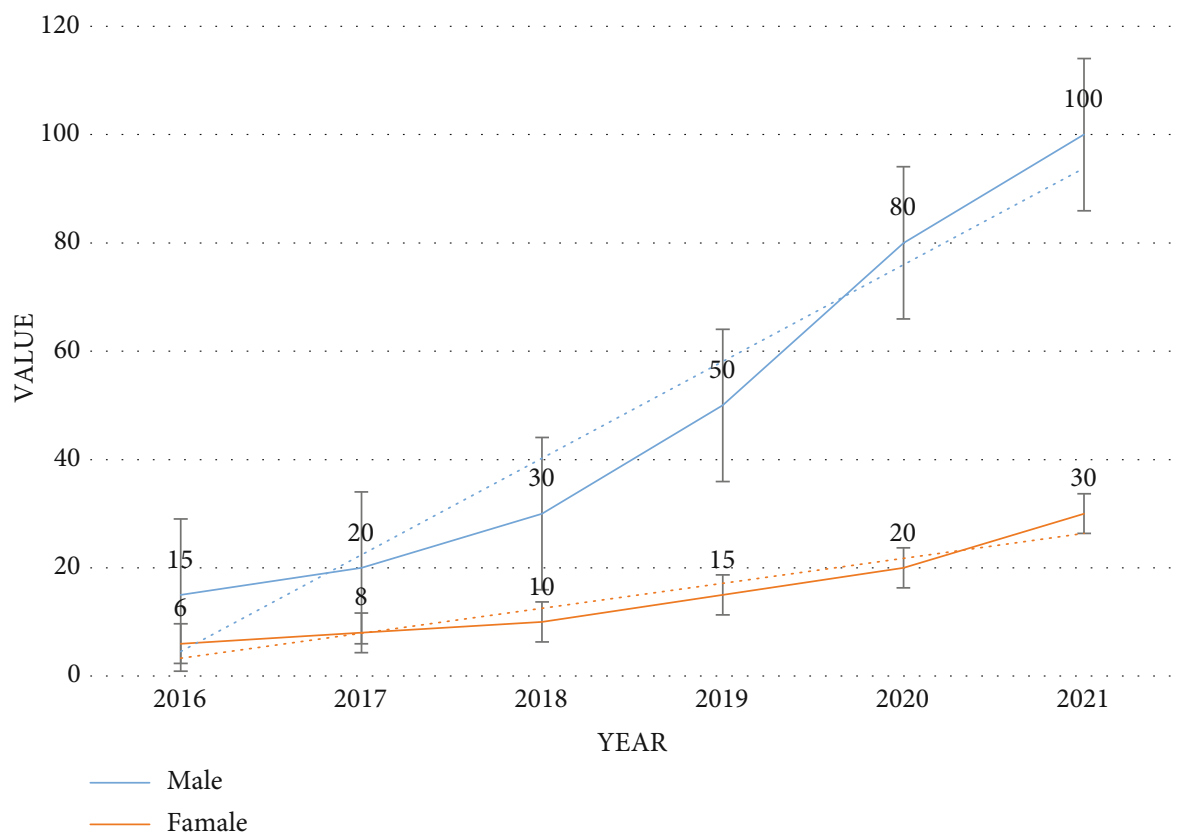

Figure 7: The total number of people in functional training in different places across the country.

TABLE 5: Selective functional action assessment content and purpose.

\begin{tabular}{|c|c|c|}
\hline Serial number & Name & Evaluation purpose \\
\hline 1 & Cervical spine assessment & $\begin{array}{c}\text { Neck spine flexion and extension degree, atlanto-occipital joint } \\
\text { flexibility, and neck } \\
\text { The degree of spinal rotation and lateral flexion }\end{array}$ \\
\hline 2 & Evaluation of upper limb movement patterns & $\begin{array}{l}\text { The entire range of shoulder movement, including internal and } \\
\text { external rotation, abduction, adduction, extension, and flexion } \\
\text { The activity of the song }\end{array}$ \\
\hline 3 & Multistep buckling assessment & Normal flexion of both hips and spine \\
\hline 4 & Multistep stretch evaluation & Normal extension of the shoulders, hips, and spine \\
\hline 5 & Standing on one foot assessment & Static and dynamic single-leg stability \\
\hline 6 & Squat with arms up head & Symmetrical flexibility of both hips, knees, and ankles \\
\hline
\end{tabular}

important. This paper conducts functional training research on the basis of multisensor information fusion. As shown in Figure 7 , the number of functional training in different parts of the country in the past 6 years has changed greatly compared with before. This makes our research very meaningful.

After the initial screening and finding out the functional actions, we can formulate different training programs for different people according to the specific results and start targeted training. If there is damage or bad actions during training it can be adjusted in time. In this case, a selective functional movement assessment (SFMA) is required to carry out strict and standardized training in the later stage according to the patient's adaptability and personal physique, as shown in Table 5:

4.2. Discussion. Multisensor information fusion technology is to use the most popular computer, $5 \mathrm{G}$, Internet of Things, and other technologies and use a variety of different sensors or information and data from a variety of different sources. Information or data is summarized and analyzed, and a detailed result is finally obtained to complete an information processing process for the final decision. There are many ways to obtain sports information. People use different ways and methods to obtain different sports information. With the support of a multisensor information fusion platform, we can go through a variety of ways. Different information sources can easily obtain a lot of sports information, so as to finally get the information you want to get. Traditional functional training is a training that teaches people how to use strength with the help of professionals. It is a complete, systematic, and planned training and functional training based on multisensor information fusion; it is more suitable for the current era than the traditional ones and can help people complete the corresponding training faster.

\section{Conclusions}

This article is a research on sports information acquisition and functional training based on multisensor information 
fusion technology. In the past, traditional ways to obtain sports information included newspapers, radio, and television. The advantage is that the information source of the traditional method is single but true and reliable. The disadvantage is that the transmission is slower than the latest information, and the efficiency is very low. Functional training is a very professional technology before, and it requires professional staff to operate. It is difficult for ordinary people to perform after being injured, so based on the functionality of multisensor information fusion technology, training can facilitate scientific and systematic training. Since the beginning of the 21st century, the multisensor information fusion technology has developed rapidly. Some people are focusing on and developing new calculation models, while others want to modify the original traditional algorithms to achieve better results. Information fusion is a development direction in the future. It will be a trend in the world in the next few years and even decades, and our research will follow the trend in the future sports information acquisition and functional training be utilized.

\section{Data Availability}

No data were used to support this study.jjj

\section{Conflicts of Interest}

The authors declare that there is no conflict of interest with any financial organizations regarding the material reported in this manuscript.

\section{Acknowledgments}

This study was funded by the fund project of the State Ethnic Affairs Commission (No. 2020-GMD-080) and the National Social Science Fund of China (No. 17CTY008).

\section{References}

[1] Z. Yu, L. Chang, and B. Qian, "A belief-rule-based model for information fusion with insufficient multi-sensor data and domain knowledge using evolutionary algorithms with operator recommendations," Soft Computing, vol. 23, no. 13, pp. 5129-5142, 2019.

[2] J. Raitoharju, S. Kiranyaz, and M. Gabbouj, “Training radial basis function neural networks for classification via classspecific clustering," IEEE Transactions on Neural Networks and Learning Systems, vol. 27, no. 12, pp. 2458-2471, 2016.

[3] C. Á. Raquel, H. C. Aurora, and L. P. Josefa, "Fusion estimation from multisensor observations with multiplicative noises and correlated random delays in transmission," Mathematics, vol. 5, no. 3, pp. 45-45, 2017.

[4] X. Zhou and P. Jiang, "Variation source identification for deep hole boring process of cutting-hard workpiece based on multisource information fusion using evidence theory," Journal of Intelligent Manufacturing, vol. 28, no. 2, pp. 255-270, 2017.

[5] M. Zhou, Y. Long, W. Zhang et al., "Adaptive genetic algorithm-aided neural network with channel state information tensor decomposition for indoor localization," IEEE Transactions on Evolutionary Computation, p. 1, 2021.
[6] P. Gong, Y. Fei, and L. Song, "Road recognition method of wheel-tracked robot based on multi-sensor information fusion," Shanghai Jiaotong Daxue Xuebao/Journal of Shanghai Jiaotong University, vol. 51, no. 4, pp. 398-402, 2017.

[7] M. Mosalanejad and M. M. Arefi, "UKF-based soft sensor design for joint estimation of chemical processes with multisensor information fusion and infrequent measurements," IET Science, Measurement \& Technology, vol. 12, no. 6, pp. 755-763, 2018.

[8] W. Bohuai, "Research on data acquisition and fusion system based on wireless sensor," Acta Technica CSAV (Ceskoslovensk Akademie Ved), vol. 62, no. 1, pp. 469-477, 2017.

[9] J. Li and C. Wang, "Research on efficient fusion methods for reliable information in wireless sensor networks," Revista de la Facultad de Ingenieria, vol. 32, no. 11, pp. 583-587, 2017.

[10] K. H. Byun, S. J. Kim, and J. W. Kwon, "Development of a multi-sensor fusion-based traffic information acquisition system with robust to environmental changes using mono camera, radar and infrared range finder," The Journal of the Korea Institute of Intelligent Transport Systems, vol. 16, no. 2, pp. 36-54, 2017.

[11] L. Zheng, R. Ji, W. Liao, and M. Li, “A positioning method for apple fruits based on image processing and information fusion," IFAC-Papers OnLine, vol. 51, no. 17, pp. 764-769, 2018.

[12] X. Xu, M. Wang, L. Luo, Z. Meng, and E. Wang, “An indoor pedestrian localization algorithm based on multi-sensor information fusion," Journal of Computer and Communications, vol. 5, no. 3, pp. 102-115, 2017.

[13] Y. Xu, L. Chen, Z. Yuan, and T. Xie, "Intelligent recognition of tool wear conditions based on the information fusion," Zhendong yu Chongji/Journal of Vibration and Shock, vol. 36, no. 21, pp. 257-264, 2017.

[14] R. Miyagusuku, A. Yamashita, and H. Asama, "Data information fusion from multiple access points for WiFi-based selflocalization," IEEE Robotics \& Automation Letters, vol. 4, no. 2, pp. 269-276, 2018.

[15] M. Zhou, Y. Li, M. J. Tahir, X. Geng, Y. Wang, and W. He, "Integrated statistical test of signal distributions and access point contributions for Wi-Fi indoor localization," IEEE Transactions on Vehicular Technology., vol. 70, no. 5, pp. 5057-5070, 2021.

[16] Q. Bi, X. Jiang, X. Liu, L. He, and T. Cheng, "Information fusion of imaging that high dynamic range in welding region based on image registration," Shanghai Jiaotong Daxue Xuebao/Journal of Shanghai Jiaotong University, vol. 50, no. 12, pp. 1915-1920, 2016.

[17] J. Yang and Q. Na, “Avoiding obstacle system of intelligent vehicle based on multi-sensor information fusion," IPPTA: Quarterly Journal of Indian Pulp and Paper Technical Association, vol. 30, no. 8, pp. 800-807, 2018.

[18] H. Yuan and G. Wu, "Fault diagnosis of transformer based on multi-information fusion," Gaoya Dianqi/High Voltage Apparatus, vol. 54, no. 9, pp. 103-110, 2018.

[19] D. Xu, M. Xing, J. Fu, and G. Sun, "Multi-aspect components extraction and fusion based on attributed scattering center model," Xi Tong Gong Cheng Yu Dian Zi Ji Shu/Systems Engineering and Electronics, vol. 39, no. 6, pp. 1197-1202, 2017.

[20] B. J. Liu, Q. W. Yang, W. U. Xiang, S. D. Fang, and F. Guo, "Application of multi-sensor information fusion in the fault 
diagnosis of hydraulic system," International Journal of Plant Engineering \& Management, vol. 22, no. 1, pp. 12-20, 2017.

[21] L. Li, L. Hui, and S. Li, "Intelligent fire monitoring system based on the information fusion algorithm," Sensor Letters, vol. 14, no. 11, pp. 1094-1098, 2016.

[22] M. Liu, J. Chen, X. Zhao, L. Wang, and Y. Tian, "Dynamic obstacle detection based on multi-sensor information fusion," IFAC-Papers OnLine, vol. 51, no. 17, pp. 861-865, 2018.

[23] X. Ouyang, W. He, W. Lu, and Z. Bian, "Study on 3D border surveillance system and multi-sensor information fusion technology," Bandaoti Guangdian/Semiconductor Optoelectronics, vol. 39, no. 2, pp. 298-304, 2018.

[24] Z. Ming, L. Yun, and H. Gang, "Multi-sensor information fusion predictive control algorithm," International Journal of Multimedia and Ubiquitous Engineering, vol. 11, no. 4, pp. 49-58, 2016.

[25] X. Zhu, T. Shi, X. Jin, and Z. Du, "Multi-sensor information fusion based control for VAV systems using thermal comfort constraints," Building Simulation, vol. 14, no. 4, pp. 10471062, 2020.

[26] L. Yue, D. K. Jha, A. Ray, and T. A. Wettergren, "Information fusion of passive sensors for detection of moving targets in dynamic environments," IEEE Transactions on Cybernetics, vol. 47, no. 1, pp. 93-104, 2016.

[27] W. Cai, S. Guo, S. Zhang, and J. Lin, "Unified framework of modeling and simulations for multi-platforms multi-sensors multi-objects source information fusion (M3SIF) system," Journal of Information Hiding and Multimedia Signal Processing, vol. 9, no. 3, pp. 760-767, 2018. 